\title{
Application of the Laser Speckle-Correlation Method for Determining the Shrinkage Vector of a Light-cured Resin
}

\author{
Tomomi SATO ${ }^{1}$, Masashi MIYAZAKI ${ }^{2}$, Akitomo RIKUTA ${ }^{2}$ and Kouichi KOBAYASHI ${ }^{3}$ \\ ${ }^{1}$ Nihon University Graduate School of Dentistry, Department of Operative Dentistry, 1-8-13, Kanda-Surugadai, Chiyoda-ku, \\ Tokyo 101-8310, Japan \\ ${ }^{2}$ Nihon University School of Dentistry, Department of Operative Dentistry and Dental Research Center, Division of \\ Biomaterials Science, 1-8-13, Kanda-Surugadai, Chiyoda-ku, Tokyo 101-8310, Japan \\ ${ }^{3}$ Toyo Seiki Seisaku-Sho Ltd., Technical Department, Funado Support Center, 1-2-6, Funado, Itabashi-ku, Tokyo 174-0041, \\ Japan \\ Corresponding author, E-mail:miyazaki-m@dent.nihon-u.ac.jp
}

Received March 29, 2004/Accepted May 11, 2004

\begin{abstract}
The purpose of this study was to develop a method to monitor the free-shrinkage vectors of a light-cured resin using laser speckle contrast measurement. The laser speckle contrast measurement system comprised a semiconductor laser, a speckle analyzer, and a computer-controlled data acquisition system. A light-cured resin was condensed into a glass tube and irradiated using a curing unit, the light intensity of which was adjusted to either 100 or $600 \mathrm{~mW} / \mathrm{cm}^{2}$. The speckle patterns obtained from the lateral surface of the specimen were monitored using a CCD camera connected to a computer. The speckle images were analyzed using a phase-only correlation algorithm, and movements of the pattern were traced. The speckle pattern revealed rapid movement almost immediately after light exposure was initiated, and the orientation of free-shrinkage vectors is dependent on light intensity. When the specimens were irradiated at the lower light intensity, the free shrinkage vectors were oriented towards the light source. On the other hand, when irradiated at the higher light intensity, the freeshrinkage vectors of these specimens tended to orient towards the bottom surface. The effectiveness of the employed method to determine free-shrinkage vectors is thus proven through the results of this study.
\end{abstract}

Key words: Laser speckle, Light-cured resin, Polymerization shrinkage

\section{INTRODUCTION}

Light-cured restorative resins undergo polymerization shrinkage during the curing process; this critical limitation can lead to postoperative sensitivity, microleakage, and secondary caries. The further consequences of polymerization shrinkage include tooth fractures and residual stresses that can be high enough to cause cusp movement ${ }^{1-5)}$. Although the marginal integrity of resin restorations can be improved using recently developed adhesive systems, a close relationship remains between polymerization shrinkage and the longevity of restorations ${ }^{6-8)}$. Several techniques for monitoring the polymerization shrinkage of restorative materials have been investigated, including dilatometer, linometer, and bonded deflection-disc methods ${ }^{9-15)}$. However, these methods provide homogeneous measures of shrinkage tendency. Novel approaches are thus needed to determine the shrinkage vector in order to verify the curing characteristics of light-cured resins.

The direction of polymerization shrinkage might affect the marginal adaptation of the restoration. It was previously suggested that light-cured resin shrinks towards the light-irradiated surface of a restoration $^{16,17)}$. As the irradiated surface was predicted to shrink faster than the deeper portions, the direction of shrinkage was expected to be oriented towards the light source. However, a more recent study using a finite elemental technique revealed that the shrinkage vector was not significantly affected by the orientation of the incoming light ${ }^{18)}$. The authors concluded that the shape of the cavity and the bonding states of the restoration predominantly determined the shrinkage direction.

Measuring the shrinkage vector during the polymerization of a light-cured resin is challenging, because shrinkage occurs rapidly after light irradiation commences. In the present study, the digital laser speckle-correlation method was used to determine the shrinkage vector of light-cured resin. The laser speckle-correlation method is a non-contact and non-destructive technique that is simple to use and does not require special surface treatment of the specimen $^{19-22)}$. The laser speckle phenomenon is observed when an intense beam of coherent light reflects off the surface of an object. The speckles are formed due to the random interference of coherent waves that are scattered by the rough surface. These speckles appear as three-dimensional diffraction fields of random intensity fluctuations if the object possesses certain physical characteristics. The contrast made by bright and dark spots can be determined from digital images recorded with a matrix charged couple device (CCD). The latter was connected to a computer that calculated the cross- 

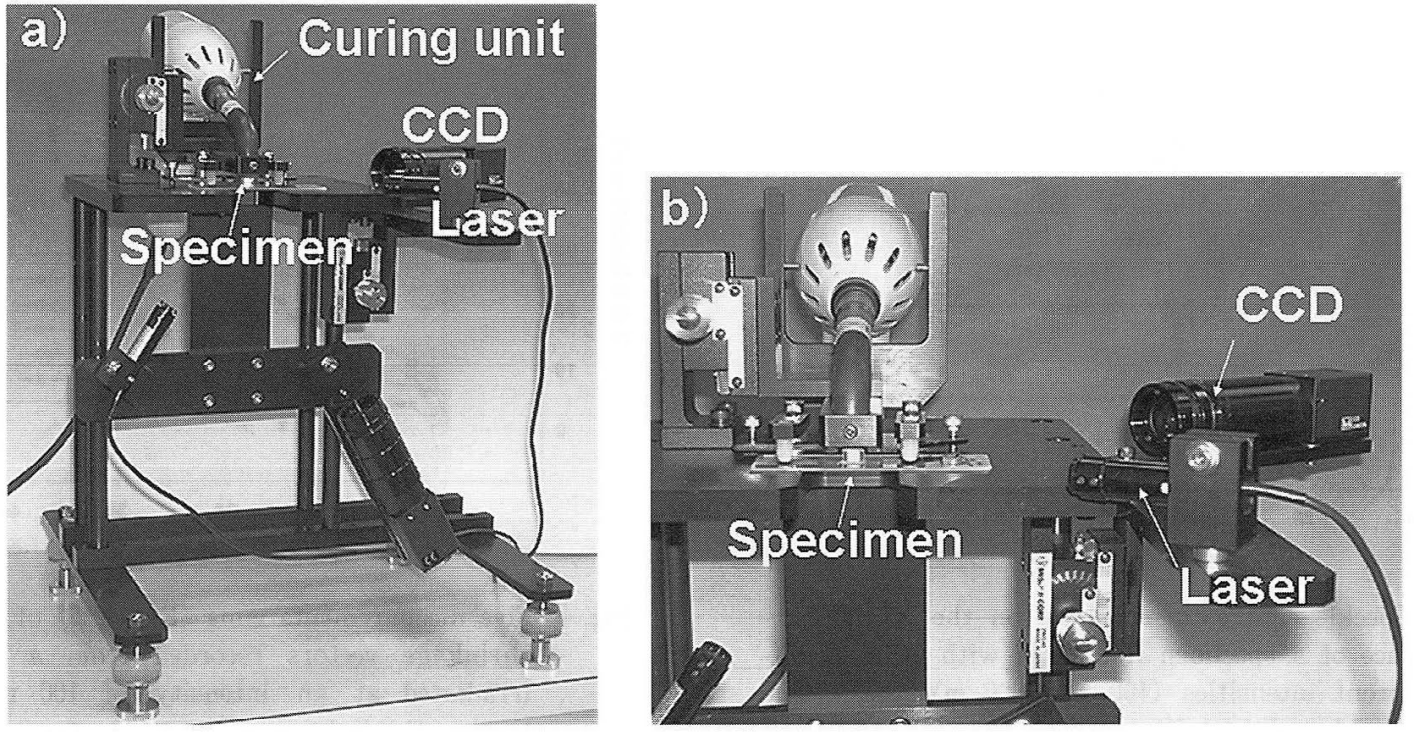

Fig. 1 a) Experimental set-up of the laser speckle-contrast measurement system.

b) The specimen was placed $20 \mathrm{~cm}$ from the laser source and the CCD camera was placed 20 $\mathrm{cm}$ from the sample surface at an angle of $30^{\circ}$ from the incident beam.

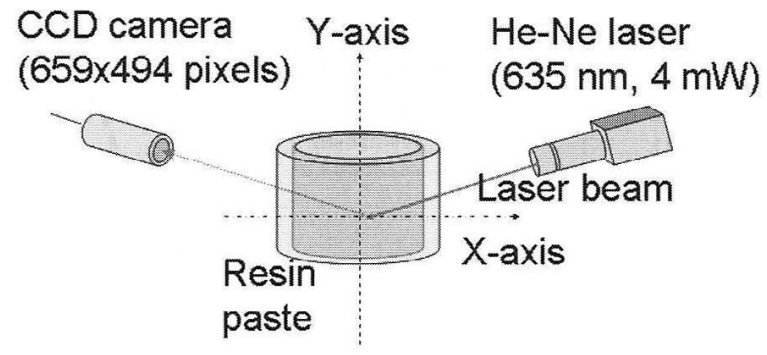

Fig. 2 Schematic diagram of the location of the laser, the CCD camera and the specimen.

correlation of images to deliver the speckle displacement ${ }^{23-26)}$. When a resin composite, or any part of it, is in motion due to the polymerization reaction, the speckle patterns that are observed reveal the movement of the resin paste.

The purpose of this study was to determine the free-shrinkage vectors of a light-cured resin using the laser speckle-correlation method. This investigation also tested the hypothesis that the laser specklecorrelation method is a useful technique for studying the polymerization characteristics of light-cured resins.

\section{MATERIALS AND METHODS}

\section{Light-cured resin and the curing unit}

A hybrid-type light-cured resin, Lite-Fil II A (Shade A3, Lot No. 100328, Shofu Inc., Kyoto, Japan), was used in this study. An Optilux 400 curing unit (Demetron/Kerr, Danbry, CT, USA) was connected to a variable transformer and the light intensity was adjusted to 100 or $600 \mathrm{~mW} / \mathrm{cm}^{2}$, as measured using a dental radiometer (Model 100, Demetron/Kerr).

Experimental setup

The test apparatus, including the laser speckle equipment (Toyo Seiki Seisaku-sho Ltd., Tokyo, Japan), is illustrated in Fig. 1. The experimental setup consisted of a coherent light source (MLXS-D13, 635 $\mathrm{nm}$, spot size $3 \times 6 \mathrm{~mm}$ with output power of $4 \mathrm{~mW}$, Kikoh Giken Co., Hyogo, Japan), a CCD camera $(659 \times 494$ pixels, CS8550-01, Tokyo Electronic Industry Co., Tokyo, Japan), and a personal computer. The resin composite was placed in a glass mold (4.0 $\mathrm{mm}$ diameter $\times 4.0-\mathrm{mm}$ height) on a glass slide (No. 5116, Muto Pure Chemicals Co., Tokyo, Japan). The specimen was placed $20 \mathrm{~cm}$ from the laser source, and the CCD camera $20 \mathrm{~cm}$ from the sample surface at an angle of $30^{\circ}$ from the incident beam (Fig. 2). The spatial resolution of this system was determined as $5 \mu \mathrm{m}$, and the analytical accuracy of the movement was calculated as $0.25 \mu \mathrm{m}$. To minimize vibration interference, the laser was fixed to a block, and the CCD camera and sample stage were placed in a darkened chamber.

A laser beam from the semiconductor laser was focused on the lateral surface of the specimen. The CCD camera was adjusted until sharply focused speckle patterns were observed on the video monitor. Light irradiation of the resin paste was performed for 30 seconds and data were collected for $120 \mathrm{sec}$ onds from the start of the irradiation. The specklecorrelation fringes were grainy patterns (compared to the true interference fringes) and had poor contrast. Therefore, a Fourier phase-only correlation algorithm $^{27)}$ was used to improve and enhance the quality of the speckle patterns. The speckle patterne 


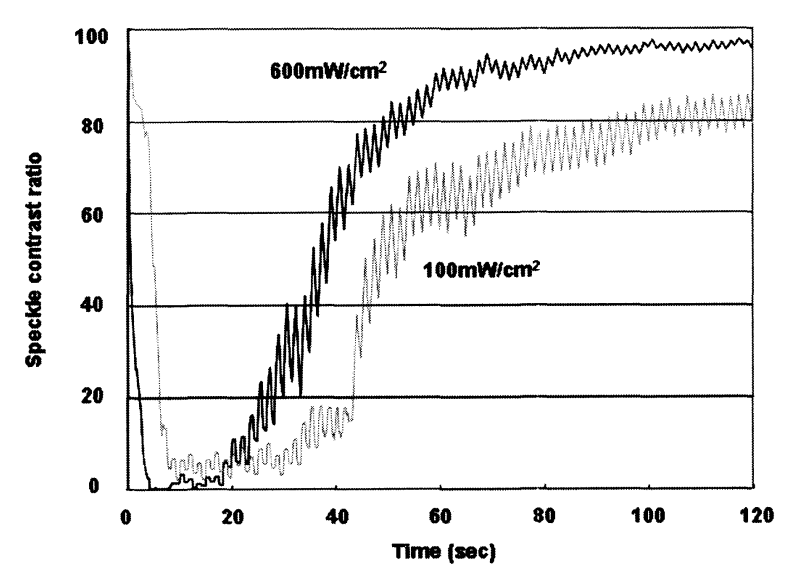

Fig. 3 Speckle contrasts measured on the lateral surface of a specimen irradiated with light at different intensities $\left(100\right.$ and $\left.600 \mathrm{~mW} / \mathrm{cm}^{2}\right)$. The speckle contrasts decreased soon after the start of light irradiation and gradually increased, particularly for the specimen irradiated at a higher light intensity.

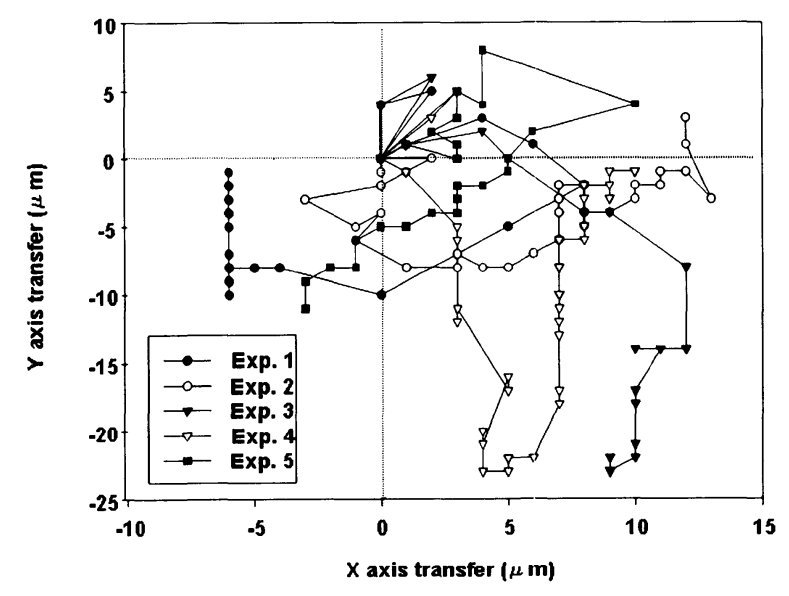

Fig. 5 Five measurements runs (Exp. 1-5) of the freeshrinkage vectors recorded from a specimen irradiated at an intensity of $600 \mathrm{~mW} / \mathrm{cm}^{2}$. The free-shrinkage vector orientations tended to orient toward the bottom surface of the specimen.

images were then examined using an image analyzer connected to a personal computer, and the speckle contrast was shown as a function of time during measurement. Finally, the free-shrinkage vectors of each specimen were expressed using two-dimensional graphs. Five measurements were performed for each light intensity.

Measurements were performed in a constanttemperature room at $23 \pm 1^{\circ} \mathrm{C}$ and $50 \pm 5 \%$ relative humidity, with illumination from one small light source.

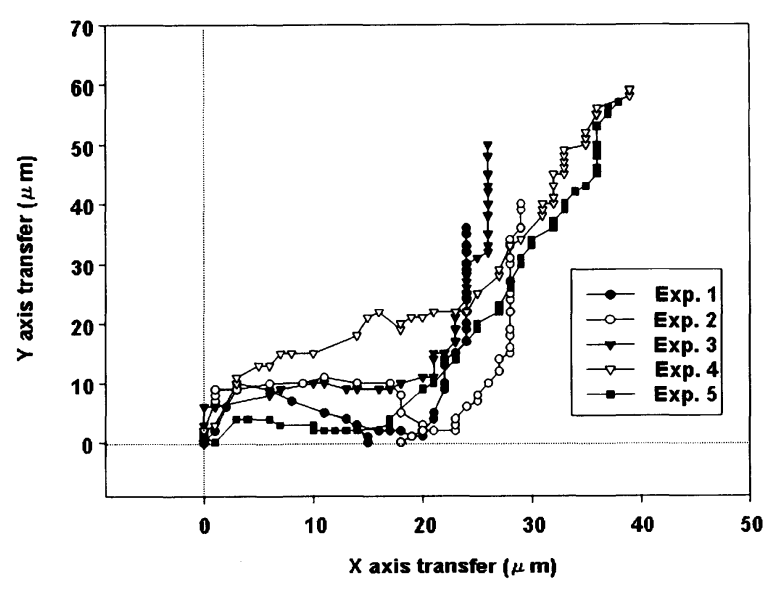

Fig. 4 Five measurements runs (Exp. 1-5) of the freeshrinkage vectors recorded from a specimen irradiated at an intensity of $100 \mathrm{~mW} / \mathrm{cm}^{2}$. During the light irradiation, the free-shrinkage vector orientations were mainly horizontal to the light source. After light irradiation, the vectors were orientated to the light source.

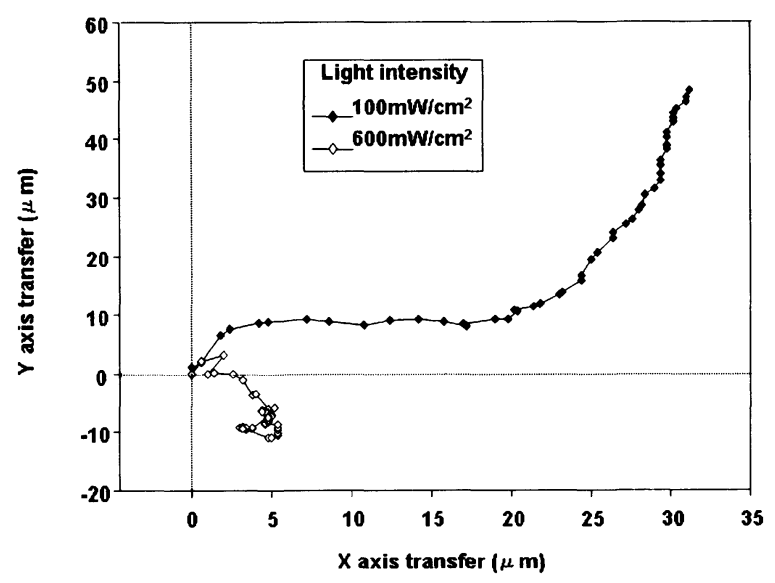

Fig. 6 Average directions of five measurement vectors recorded using different light intensities. The free-shrinkage vectors of different light intensities were different and the total displacements were much smaller than those obtained under lower light intensity.

\section{RESULTS}

The changes in speckle contrasts of the light-cured resin polymerized with different light intensities are shown in Fig. 3. The overall magnitude of the speckle contrasts decreased soon after the initial light exposure and gradually increased thereafter. The rapid response of the speckle contrasts corresponded to the polymerization reaction status in the lightcured resin. This tendency was more pronounced in the specimen that was irradiated at a higher light intensity.

Five measurements for different light intensities 
were carried out, and the directions of the freeshrinkage vectors recorded are shown in Figs. 4 and 5. For each light intensity, displacements of the speckle patterns were recorded every 2.5 seconds, and the values were plotted for five different measurements. The average displacements of the five measurements for two different light intensities are illustrated in Fig. 6.

When the specimens were irradiated at the lower light intensity, the directions of the free-shrinkage vectors were divided into two phases. Initially, the free-shrinkage vector orientations were mainly horizontal to the light source; whereas after light irradiation, the free-shrinkage vectors were oriented towards the light-irradiated surface.

Specimens irradiated at the higher light intensity showed different free-shrinkage vectors from those exposed to the lower intensity light. From the start of the irradiation, the free-shrinkage vectors of these specimens tended to orient towards the bottom surface, and the total displacements were much smaller than those obtained under the lower light intensity.

\section{DISCUSSION}

This is not the first speckle-interferometric technique that has been used to observe the shrinkage of resin composites. Digital interferometric methods have previously been used to monitor the linear polymerization shrinkage of commercially available lightcured resins ${ }^{28,29)}$. These studies have shown that the interferometry method is a valuable technique for obtaining shrinkage measurements, which has the potential for improved accuracy compared with alternative methods that require considerable temperature corrections and data calibrations. Digital interferometry does not need calibration because the wavelength of the laser light source provides an absolute length standard. When the resin paste was light polymerized, the test area moved and the coherent wave fronts were scattered, resulting in a change in the speckle patterns. The speckle patterns were imaged on the CCD camera and stored in its memory. The digital absolute-value subtraction between the image of the object in its initial state and the images taken during polymerization resulted in an image with speckle pattern displacement $(\Delta d)$ that can be expressed as follows:

$$
\Delta d=n \lambda / 2 \sin \alpha
$$

Here, $\lambda$ is the wavelength of the laser light, $n$ is the number of fringes forming the interferogram, and $\alpha$ is the angle of inclination of the coherent wave front to the surface. The speckle patterns have a similar appearance to Moire fringes and represent contours of equal displacement, with each fringe resulting from a displacement of the order of one-half of the wavelength of the laser light source used. The laser speckle-contrast measurement technique has the added advantage that a small amount of sample can be analyzed with great accuracy $(<1 \mu \mathrm{m})^{20)}$.

During speckle-contrast measurement, the surface of the resin paste moves as a result of the polymerization reaction, leading to changes in the speckle patterns. The contrast of a speckle pattern can be determined using digital imaging. This provides a method for observing the properties of a system independently of the total scattering intensity. When the object of interest is in motion, the observed speckle field reflects this motion. In the present study, lower numbers of speckle contrast indicated that the movement of the resin composite during polymerization occurred rapidly. The majority of the polymerization shrinkage occurred during and immediately after irradiation, which corresponded to the lower number of speckle contrasts measured during irradiation (Fig. 2).

The free-shrinkage vectors determined by the speckle contrast measurement indicated that the orientation of the polymerization reaction differed within the resin paste. Most light-cured materials use camphorquinone as a photoinitiator ${ }^{30,31)}$, and an adequate intensity of visible light of wavelength around $470 \mathrm{~nm}$ is required to initiate polymerization $^{32,33)}$. Light of the appropriate wavelength is absorbed by the photosensitizer, which then reacts in its excited state with an amine-reducing agent to produce reactive free radicals ${ }^{34,35)}$. The transmission of light through the material and the composition of the photoinitiator both influence the mechanical properties of light-cured materials ${ }^{36)}$. The intensity of light passing through a material is controlled by both the absorption by and scattering of its components ${ }^{37,38)}$. The rate of polymerization is reduced at lower light intensities, which results in a lower shrinkage rate ${ }^{39-41)}$. In the present study, differences in light intensity within the resin paste resulted in a slower polymerization reaction in the bottom layer. If the light intensity was low enough to create a gradient in polymerization velocity within the lightcured resin, the direction of the free-shrinkage vectors would be oriented towards the light (Fig.4). However, if the entire resin paste was polymerized at a higher light intensity, the shrinkage vectors would be similar to those observed in self-cured resins (Fig. 5).

It was previously reported that $233 \mathrm{~mW} / \mathrm{cm}^{2}$ was the lowest light intensity capable of curing resins at a depth of $2 \mathrm{~mm}^{42)}$. The results of the present study showed that a light intensity of $100 \mathrm{~mW} / \mathrm{cm}^{2}$ induced a greater displacement of the resin paste in the direction of the light-irradiated surface. This can be explained in terms of the initiation and propagation steps of the polymerization process. Resin paste has been reported to take a relatively long time to reach its gel point when exposed to low light intensities ${ }^{43)}$. 
This might have a role in creating differences in the polymerization reaction within the resin paste. By contrast, no such differences were seen within resin pastes that were irradiated with higher light intensity. A number of factors can influence shrinkage vectors, including the adherence of resin paste to the cavity floor, the configuration factor of the restoration, the elastic modulus and flow of the resin paste, and the intensity of the curing unit. Further research is needed to clarify the specific effects of these factors on polymerization characteristics in order to determine their clinical implications.

Resin composites show viscous flow characteristics prior to hardening ${ }^{44,45)}$. The gelation of a resin composite is identified as the time when the polymer chain exhibits an infinite molecular weight due to the cross-linking of monomers. It is not clear when this transition occurs because of the significant viscoelastic behavior of the polymer structure ${ }^{46)}$. The results of the present study revealed that the resin pastes moved rapidly after light irradiation commenced (Fig. 3). At the higher light intensity, the polymer network seemed to be too cross-linked to permit movement of the monomers. By contrast, at the lower light intensity, the majority of the displacements occurred after irradiation had ceased (Fig. 6). This phenomenon might be related to a decrease in the contraction strain of the light-cured resin produced by irradiation at a lower light intensity, hence allowing stress relaxation to occur. A slower curing process might therefore allow stress relaxation to take place during polymerization, leading to better marginal adaptation of the restoration. On the other hand, other factors that contribute to the mechanical properties of light-cured materials, such as type of filler ${ }^{47-49)}$ and initiator system, ${ }^{34,35,50)}$ should be considered.

Although there are many published data on volumetric shrinkage characteristics ${ }^{9-15)}$, there is lack of clear understanding on the shrinkage vectors of light-cured resins. It is not the purpose of the present work to list and compare all the methods of shrinkage characterization, but to introduce the utility of the laser speckle contrast measurement as a research tool. Though this experimental technique is a suitable tool for monitoring the shrinkage vectors of light-cured resins, further investigations should be carried out to observe the three-dimensional changes of polymerization processes.

In conclusion, this study investigated the laser speckle correlation method for characterizing the polymerization reaction of a light-cured resin. Changes in the speckle patterns were correlated with light intensity, which provided information on the status of the polymerization reaction. This approach represents an alternative method for determining the freeshrinkage vectors of light-cured resins, and has improved accuracy and precision when compared with existing techniques ${ }^{28,29)}$.

\section{ACKNOWLEDGEMENTS}

This work was supported, in part, by a grant from the Dental Research Center, Nihon University School of Dentistry, Japan (2003, 2004), from the Ministry of Education, Culture, Sports, Science, and Technology of Japan to promote multi-disciplinary research project, and Grant-in Aid for Encouragement of Young Scientists (B) 40287660 from the Japan Society for the Promotion of Science. The authors would like to thank Dr. Toshiki Ooguro and Ms. Naoko Noguchi for their contributions to the experiments.

\section{REFERENCES}

1) Tani $Y$, Nambu T, Ishikawa A, Katsuyama S. Polymerization shrinkage and contraction force of composite resin restorative inserted with "Megafiller". Dent Mater J 1993; 12: 182-189.

2) Ikemi T, Nemoto K. Effects of lining materials on the composite resins shrinkage stresses. Dent Mater $J$ 1994; 13: 1-8.

3) Kinomoto Y, Torii M. Photoelastic analysis of polymerization contraction stresses in resin composite restorations. J Dent 1998; 26: 165-171.

4) Winkler MM, Katona TR, Paydar NH. Finite element stress analysis of three filling techniques for class $\mathrm{V}$ light-cured composite restorations. J Dent Res 1996; 75: $1477-1483$.

5) Irie M, Suzuki K, Watts DC. Marginal gap formation of light-activated restorative materials: effects of immediate setting shrinkage and bond strength. Dent Mater 2002; 18: 203-210.

6) Han L, Edward C, Okamoto A, Iwaku M. A comparative study of fluoride-releasing adhesive resin materials. Dent Mater J 2002; 21: 9-19.

7) Inoue S, Murata Y, Sano H, Kashiwada T. Effect of $\mathrm{NaOCl}$ treatment on bond strength between indirect resin core-buildup and dentin. Dent Mater J 2002; 21: 343-354.

8) Han L, Okamoto A, Ishikawa K, Iwaku M. EPMA observation between dentin and resin interfaces. Part 1 . Comparison of wet and dry technique after short-term stored in water. Dent Mater J 2003; 22: 115-125.

9) Walls AWG, McCabe JF, Murray JJ. The polymerization contraction of visible-light activated composite resins. J Dent 1988; 16: 177-181.

10) Rees JS, Jacobsen PH. The polymerization shrinkage of composite resins. Dent Mater 1989; 5: 41-44.

11) Watts DC, Cash AJ. Determination of polymerization shrinkage kinetics in visible-light-cured materials: methods development. Dent Mater 1991; 7: 281-287.

12) Iga $M$, Takeshige $F$, Ui $T$, Torii $M$, Tsuchitani $Y$. The relationship between polymerization shrinkage measured by a modified dilatometer and the inorganic filler content of light-cured composites. Dent Mater J 1991; 10: $38-45$.

13) Lai JH, Johnsin AE. Measuring polymerization 
shrinkage of photo-activated restorative materials by a water-filled dilatometer. Dent Mater 1993; 9: 139-143.

14) de Gee AJ, Feilzer AJ, Davidson CL. True linear polymerization shrinkage of unfilled resins and composites determined with a linometer. Dent Mater 1993; 9: 11-14.

15) Fano V, Ortalli I, Pizzi S, Bonanini M. Polymerization shrinkage of microfilled composites determined by laser beam scanning. Biomaterials 1997; 18: 467-470.

16) Hansen EK. Visible light-cured composite resins: polymerization contraction, contraction pattern and hygroscopic expansion. Scand J Dent Res 1982; 90: 329-335.

17) Lutz F, Krejci I, Luescher B, Oldenburg TR. Improved proximal margin adaptation of Class II composite resin restorations by use of light-reflecting wedges. Quintessence Int 1986; 17: 659-664.

18) Versluis A, Tantbirojn D, Douglas WH. Do dental composites always shrink toward the light? J Dent Res 1998; 77: 1435-1445.

19) Brillaud J, Lagattu F. Limits and possibilities of laser speckle and white-light image-correlation methods: theory and experiments. Appl Opt 2002; 41: 6603-6613.

20) Yamaguchi I. Holography, speckle, and computers. Opt Lasers Eng 2003; 39: 411-429.

21) Mohan NK, Rastogi P. Recent developments in digital speckle pattern interferometry. Opt Lasers Eng 2003; 40: $439-445$.

22) Jones J, Tyrer J. An introduction to laser speckle strain imaging as a quantitative tool for structural NDT and lifetime prediction. Insight 2003; 45: 272-275.

23) Román JF, Fernández P, Moreno V, Abeleira M, Gallas M, Suárez D. The mechanical behavior of human mandibles studied by electronic speckle pattern interferometry. Eur J Orthod 1999; 21: 413-421.

24) Fukuoka S, Hotokebuchi T, Jingushi S, Fujii $H$, Sugioka Y, Iwamoto Y. Evaluation of blood flow within the subchondral bone of the femoral head: use of the laser speckle method at surgery for osteonecrosis. J Orthop Res 1999; 17: 80-87.

25) Kishen A, Murukeshan VM, Krishnakumar V, Asundi A. Analysis on the nature of thermally induced deformation in human dentine by electronic speckle pattern interferometry (ESPI). J Dent 2001; 29: 531-537.

26) Ayers MR, Hunt AJ. Observation of the aggregation behavior of silica sols using laser speckle contrast measurements. J Non-Cryst Solids 2001; 290: 122-128.

27) Kobayashi K, Nakajima H, Aoki T, Kawamata M, Higuchi T. Principles of phase only correlation and its applications. ITE Technical Report 1996; 20: 1-6.

28) Fogleman EA, Kelly MT, Grubbs WT. Laser interferometric method for measuring linear polymerization shrinkage in light cured dental restoratives. Dent Mater 2002; 18: 324-330.

29) Demoli N, Knežević A, Tarle Z, Meniga A, Šutalo J, Pichler G. Digital interferometry for measuring of the resin composite thickness variation during blue light polymerization. Opt Commun 2004; 231: 45-51.

30) Taira M, Urabe H, Hirose T, Wakasa K, Yamaki M. Analysis of photoinitiators in visible-light-cured dental composite resins. J Dent Res 1988; 67: 24-28.

31) Hayakawa T, Kikutake K, Nemoto K. Effectiveness of the addition of water-soluble photoinitiator into the self-etching primers on the adhesion of a resin composite to polished dentin and enamel. Dent Mater J 1999; 18: $324-333$.

32) Nomoto R. Effect of light wavelength on polymerization of light-cured resins. Dent Mater J 1997; 16: 6073 .

33) Nomoto R, Hirasawa T. Evaluation of the amount of residual monomer on UDMA-based resins by FTIR. Dent Mater J 1999; 18: 176-183.

34) Ikemura K, Kouro Y, Endo T. Effect of 4acryloxyethyltrimellitic acid in a self-etching primer on bonding to ground dentin. Dent Mater J 1996; 15: 132-143.

35) Nyunt MM, Imai Y. Adhesion to dentin with resin using sulfinic acid initiator system. Dent Mater $J$ 1996; 15: 175-182.

36) Cook WD. Photopolymerization kinetics of dimethylacrylates using the camphorquinone/amine initiator system. Polymer 1992; 33: 600-609.

37) Arikawa $\mathrm{H}$, Fujii $\mathrm{K}$, Kanie $\mathrm{T}$, Inoue $\mathrm{K}$. Light transmittance characteristics of light-cured composite resins. Dent Mater 1998; 14: 405-411.

38) Arikawa H, Kanie T, Fujii K, Shinohara N, Takahashi $\mathrm{H}$, Inoue $\mathrm{K}$. A method for evaluating color stability of light-cured composite resins using an experimental filter. Dent Mater J 2000; 19: 338-345.

39) Watanabe K, Ohnishi E, Kaneshima T, Mine A, Yatani $\mathrm{H}$. Porcelain veneer bonding to enamel with plasma-arc light resin curing. Dent Mater J 2002; 21: 61-68.

40) Arikawa H, Kanie T, Fujii K, Fukui K, Homma T. Mechanical properties of light-cured composite resins cured through filters that simulate enamel. Dent Mater J 2002; 21: 147-155.

41) Okamoto M, Mine A, Watanabe K, Kawahara D, Yatani $H$. Porcelain veneer bonding to dentin and the curing performance of plasma-arc light with respect to porcelain thickness. Dent Mater J 2003; 22: 313-320.

42) Rueggeberg FA, Caughman WF, Curtis JW Jr. Effect of light intensity and exposure duration on cure of resin composite. Oper Dent 1994; 19: 26-32.

43) Sakaguchi RL, Berge HX. Reduced light energy density decreases post-gel contraction while maintaining degree of conversion in composites. J Dent 1998; 26: 695-700.

44) Feilzer AJ, De Gee AJ, Davidson CL. Quantitative determination of stress reduction by flow in composite restorations. Dent Mater 1990; 6: 167-171.

45) Uno S, Shimokobe H. Contraction stress and marginal adaptation of composite restorations in dentinal cavity. Dent Mater J 1994; 13: 19-24.

46) Dauvillier BS, Aarnts MP, Feilzer AJ. Modeling of the viscoelastic behavior of dental light-activated resin composites during curing. Dent Mater 2003; 19: 277285.

47) Kakuta K, Urapepon S, Miyagawa $Y$, Ogura H, Yamanaka M, Suchatlampong C, Rittapai A. Development of metal-resin composite restorative material. 
Part 4. Flexural strength and flexural modulus of metal-resin composite using Ag-In alloy particles as filler. Dent Mater J 2002; 21: 181-190.

48) Yoshida Y, Shirai K, Shintani H, Okazaki M, Suzuki K, Van Meerbeek B. Effect of presilanization filler decontamination on aesthetics and degradation resistance of resin composites. Dent Mater J 2002; 21: 383-395.

49) Urapepon S, Kakuta K, Ogura H. Development of metal-resin composite restorative material. Part 5: Evaluation of the bonding between Ag-Sn particle and 4-META coupling agent of the metal-resin composite. Dent Mater J 2003; 22: 137-145.

50) Hirabayashi C, Imai Y. Studies on MMA-TBB resin. I. Comparison of TBB and other initiators in the polymerization of PMMA/MMA resin. Dent Mater J 2002; 21: $314-321$. 